\title{
SINGLE STEP VS SERIAL DILATATION FOR TRACT CREATION IN PERCUTANEOUS NEPHROLITHOTOMY: A RANDOMIZED CONTROLLED TRIAL
}

\author{
${ }^{1}$ Senior Assistant Professor, Department of Urology, Government Stanley Hospital, Chennai. \\ ${ }^{2}$ Senior Assistant Professor, Department of Urology, Government Stanley Hospital, Chennai. \\ 3 Professor, Department of Urology, Government Stanley Hospital, Chennai. \\ ${ }^{4}$ Professor, Department of Urology, Government Stanley Hospital, Chennai. \\ 5Junior Resident, Department of Urology, Government Stanley Hospital, Chennai. \\ 6Junior Resident, Department of Urology, Government Stanley Hospital, Chennai. \\ 7Junior Resident, Department of Urology, Government Stanley Hospital, Chennai.
}

Thiruvarul P. $V^{1}$, Periasamy P2, Pitchai Balashanmugam $K^{3}$, Govindarajan P4, Lakshminarayan K. R ${ }^{5}$, Seetharam Bhat ${ }^{6}$, Thilak $^{7}$

ABSTRACT: OBJECTIVES: Percutaneous nephrolithotomy is one of the procedures of choice for renal calculi, and there are several techniques for tract dilatation. However there is a paucity in Indian literature on single dilatation techniques. This study aims to analyse the feasibility and morbidity of single step acute dilatation and to compare it with Amplatz sequential dilatation technique, for tract creation in percutaneous nephrolithotomy.

MATERIALS \& METHODS: This study was a randomized controlled trial conducted in Urology department of Govt Stanley Hospital between August 2014 and March 2015. 60 patients satisfying the inclusion criteria were selected and were randomized into two groups of 30 patients each, and for tract creation during percutaneous nephrolithotomy, serial dilatations were done in one group A and single dilatation was done in group B. X-ray exposure time, blood loss, post-operative complications, duration of stay and need for ancillary procedures like ESWL were taken into account to measure the outcome.

STATISTICAL ANALYSIS: Analysis was done using Chi-Square and Fisher's Exact tests.

RESULTS: Mean X-ray Exposure Time was 92.9 seconds in group A, and 29.43 seconds in group B. 4 patients in group A and 2 Patients in group B required transfusions. Mean hospital stay was 4.5 days in group A and 4.3 days in group B. 3 patients in group A and 2 patients in group B, required ESWL.

CONCLUSION: X-ray exposure was significantly less in single step acute dilatation technique, while there was no significant difference in other outcome measures, between the two groups.

KEYWORDS: PCNL, Single Step Dilatation, Tract Creation.

HOW TO CITE THIS ARTICLE: Thiruvarul P. V, Periasamy P, Pitchai Balashanmugam K, Govindarajan P, Lakshminarayan K. R, Seetharam Bhat, Thilak. "Single Step Vs Serial Dilatation for Tract Creation in Percutaneous Nephrolithotomy: A Randomized Controlled Trial". Journal of Evolution of Medical and Dental Sciences 2015; Vol. 4, Issue 91, November 12; Page: 15669-15672, DOI: $10.14260 /$ jemds/2015/2254.

INTRODUCTION: Percutaneous nephrolithotomy has become a surgery of choice for renal stone 2 . For improving the access, either by Amplatz sequential fascial dilators or metal telescopic dilators and to further trying to decrease the time for radiation exposure and also to decrease complication various novel approaches have been proposed.1,2

Single-step access can reduce the chance of access failure, decrease the duration of surgery and decrease the time for radiation exposure. Lack of sufficient investigation with large enough sample size, it has not become universal.

This study had been said first by Frattini and associates. ${ }^{1}$ In this they study in detail about the feasibility option for performing one-stage tract dilation method. This method is done by one-step dilation with a 24 or $28 \mathrm{~F}$ Amplatz dilator for

Financial or Other, Competing Interest: None.

Submission 21-10-2015, Peer Review 22-10-2015,

Acceptance 30-10-2015, Published 11-11-2015.

Corresponding Author:

Dr. Thiruvarul P. V,

Department of Urology,

Do. No. D-18, PA Towers Halls Road, Egmore,

Chennai-600008.

E-mail: thiruvarulpv@gmail.com

DOI:10.14260/jemds/2015/2254. the nephrostomy tract in all adult patients planned for percutaneous nephrolithotomy surgery.

AIM: To analyse the feasibility and also the morbidity in singlestep acute dilation, named "One-shot" technique and also to compare it with Amplatz sequential fascial dilatation technique regarding creation of tract in performing percutaneous nephrolithotomy.

MATERIALS AND METHODS: It is prospective randomized clinical trial, including 60 patients who underwent percutaneous nephrolithotomy surgery for the stone disease performed by one surgeon in our hospital from January 2014 to Feb 2015. Inclusion criteria included all patients who were candidate for PCNL. Then patient were randomly divided according to the dilatation technique into two groups.

In group A, sequential fascial Amplatz dilation technique was used. In group B, single step acute dilation technique was used. Two groups were similar in age, sex, stone location and stone burden.

Demographic data for all patients Haemoglobin was done before and after surgery, Functional assessment of the kidney and stone burden was defined as the maximum diameter of the stone on kidney radiograph or CT or IVU. 
Intraoperative information work up includes X-ray exposure time, (Number of seconds of X-ray exposure elapsed from the insertion of needle to placement of the Amplatz sheath).

Single step dilation technique: after anaesthesia, positioning \& calyceal needle puncture, guide wire was inserted and after dilation with screw dilator, a $24 \mathrm{~F}$ or $28 \mathrm{~F}$ Amplatz dilator followed by insertion of $26 \mathrm{~F}$ or $30 \mathrm{~F}$ Amplatz sheath depending on the stone size. Amplatz dilators with the central guide rod are removed after confirming that sheath is in the collecting system.

Considering bleeding as a complication. After becoming severe enough leading to procedural termination or requiring blood transfusion.

Post-operative complications and hospital stay were evaluated.

Statistical analysis was performed using chi-square test, Fisher's exact test.

ETHICS: The study was cleared by Ethical Committee of Govt. Stanley Medical College and Hospital, in Dec 2013.

RESULTS: Out of 60 patients, 30 patients were in each group A and B. In group A, sequential fascial Amplatz dilation technique was used. In group B, single step acute dilation technique was used.

The variable included are demographic data, intra operative details such as Technique, X-ray exposure time, Blood loss and Transfusion, Post-operative complications and hospital stay were evaluated.

Statistical analysis showed that X-ray exposure time was significantly shorter in group B compared with group A with a $p$ value $<0.001$ suggestive of highly significant.

No significant difference in bleeding complications in the two groups and $\mathrm{P}$ Value also confirmed it.

In about six patients ( 4 in group A and 2 in group B) required blood transfusion.

There was no significant difference in the hospital stay and bleeding complication in the two groups and P Value also confirmed it.

About five patients (2in group A and 3 in group B) had urinary tract infection treated conservatively with appropriate antibiotics.

About five patients ( 3 in group A and 2 in group B), complete clearance could not be obtained, requiring ancillary procedure such as ESWL.

But there was no significant difference in complication and requirement of ancillary procedure. P-Value was found to be insignificant.

There were no failures in either group to successfully enter the calyx. We did not find any difficulties in tract dilatation in either technique.

DISCUSSION: Standard option for the management of renal stones in patient who are not ideal for ESWL is Percutaneous Nephrolithotomy. PCNL tract creations are usually done by one of three dilatation technique like Alken Metal telescopic dilators, Amplatz Incremental dilator set or Balloon dilator. ${ }^{1}$

The tract dilatation step in PCNL is very vital because this can result in bleeding so selecting an appropriate dilator system is required. ${ }^{1}$
Davidoff and associate in their study showed that by using balloon dilator they found that the chance of renal haemorrhage was less. ${ }^{4}$

Balloon dilator has many advantages like one step dilation of the tract and also helps in renal displacement prevention throughout tract creation. But because of the high cost, routinely it cannot be used and it also found to have failure rate of $17 \%$, rate and it increase up to $25 \%$ in patient in whom there was previous renal surgery, other studies had also confirmed. 6

The tract dilation can also be performed with the help of Amplatz dilator or with the Alken metal dilator systems. ${ }^{1}$ and also as an alternative to failure with balloon dilation system. But their main problem is incremental nature resulting in prolongation of time in access, increase in exposure of radiation and also increases chances of the tract displacement.

In following years, a few of them trying in defining a possible system like the one-step dilation of the tract and the method when proposed it must be simple to use and also should be inexpensive, should be suited for all patients with minimizing complication like bleeding.7,8

Innovative system was developed by Goharderakhshan and colleagues. ${ }^{9}$ was named as "Radially expanding single-step nephrostomy system (RESN) system" and it was in 2001they were reporting the initial clinical result.

It has a woven sleeve of size 8Fr with inner stylet which is used in passing to desired calyx over an already placed working guide wire and tapered fascial dilator of size $30 \mathrm{Fr}$ fitted with Amplatz sheath is passed for creating the nephrostomy tract by a one step.

It was found transmitting the lesser axial force to a target when compared to that of balloon and Amplatz dilators. ${ }^{4}$ By this method, also helps in preventing the forward untoward kidney migration and radial force generation and ability for the tract dilation is the same as other dilator system. It was in 1998 Jackman and co-workers. ${ }^{10}$ in seven paediatric patients used an $11 \mathrm{~F}$ device had results found desirable and proposing it as "Mini-PERC".

Pathak and Bellman. ${ }^{6}$ used a pathway for the access sheath, had a balloon dilator for tract and containing an Amplatz-like access sheath which was found over it. Study of this device in 11 patients was found to be very positive in term of reducing the time for an access and also the blood loss, but even with their encouraging results, it was not applicable in patient with previous renal scar and also of its high cost. ${ }^{5}$ The novel one-shot technique by Frattini and colleagues. ${ }^{1}$ consist of the one step dilation of the nephrostomy tract which was rapid by using a 30F Amplatz dilator. They were trying it in about 26 patients. They analysed the parameters, like the exposure to radiation, loss of blood, and usage costs were compared with other to groups containing of the PCNL-patients were the Alken metal telescopic dilator and then also with balloon dilator group. ${ }^{1}$

They stated that one-step dilation is found to be more feasible and also to be safer, also found to be consuming less time, the cost also found to be comparatively less. But they found that their study lack an adequate number of the patients and the technique were not tried in a patient who had the renal surgery previously. ${ }^{1}$ 
The study which was by conducted by Ziae et al they evaluated the one-stage technique in around 50 adult patients 8 and compared with gradual dilation techniques. ${ }^{1}$ they concluded their study by saying that this technique can be used as primary choice in adult patients for the PCNL tract dilation. ${ }^{1}$

Our study also we found that lower exposure time to Xray in group $B$ was $29.43 \mathrm{sec}$ mean, where one-shot technique was used compared to group A was $92.90 \mathrm{sec}$ mean. P value was $<0.001$, which is highly significant. This finding correlates with above mentioned studies.

By using appropriating vector force application is important for applying in the one-step dilation of the tract. The kidney migration chance and displacement of the guide wire for creating the tract was decreased by rotational movements with sheath for advancing gradually. ${ }^{2}$

In One-shot technique the rate of guide wire and kidney displacement are low by means of lower axial force generation. This along with the consistency of the dilator explains difference in the failure rates significantly of the balloon dilator technique. ${ }^{31,4}$ when related with one-step dilation.

We found that one-shot technique could be safe, effective and also preventing from less radiation exposure. In the same way Ajmal et al. ${ }^{3}$ ziaee et al. ${ }^{4}$ Falahatkar et al. ${ }^{10}$ Sofikerim et al. ${ }^{5}$ Trevis DG et al. ${ }^{4}$ reported that one-shot technique was safe and with less radiation exposure.

Our bleeding complication and decrease in haemoglobin was managed by means of conservative therapy and blood transfusion. We found no difference between two groups.

Our study demonstrated that one-shot technique can be used for access to almost every calix. In the trained hands, we found that it was found to be effective in approaching for the "Difficult calices" also. Physical properties of the technique are found with the less axial force in comparison with that of the radial force, for a timely manner.

We found that there was very less radiation exposure and also a low rate of guide wire displacement and less chance of false passage creation.

Also we found that the technique may be requiring more experience than that of the other conventional methods, but the curve of learning is found comparatively to be smooth.

CONCLUSION: In One-shot technique, there were no failures in either group to successfully enter the calyx.

Exposure time to X-ray is significantly lower in the single step acute dilation technique, thereby decreasing the complications due to radiation exposure. P value was found to be highly significant.

We found no significant difference in bleeding complications, ancillary procedure such as ESWL and the hospital. ${ }^{1}$ stay in the two groups. $\mathrm{P}$ value was found not significant.

This Single step acute dilation technique ('One-shot' dilatation technique) was proved to be safe and effective when compared with the standard Amplatz sequential fascial dilatation technique. that single-step acute dilation, named "One-shot" technique as safe and effective like Amplatz sequential fascial dilatation technique regarding creation of tract in performing percutaneous nephrolithotomy.

\section{REFERENCES:}

1. Frattini A, Barbieri A, Salsi P. One shot: a novel method to dilate the nephrostomy access for percutaneous lithotripsy. Journal of endourology/Endourological Society. 15(9):919-23. 2001.

2. McDougall EM, Liatsikos EM, Dinlenc CZ, Smith AD: Percutaneous Approaches to the Upper Urinary Tract. In: Campbell's Urology, 8th ed., Walsh PC et al. (eds.), Philadelphia, WB Saunders, 2002; pp. 3337-40.

3. Amjadi M, Zolfaghari A, Elahian A, Tavoosi A. Percutaneous nephrolithotomy in patients with previous open nephrolithotomy: one-shot versus telescopic technique for tract dilatation. J Endourol. 2008;22(3):423-5.

4. Travis DG, Tan HL, Webb DR. Single-increment dilatation for percutaneous renal surgery: an experimental study. British journal of urology. 68(2):144-7. 1991.

5. Ziaee SA, Karami H, Aminsharifi A, Mehrabi S, Zand S, Javaherforooshzadeh A. One-stage tract dilation for percutaneous nephrolithotomy: is it justified? Journal of endourology/Endourological Society. 21(12):1415-20. 2007.

6. Joel AB, Rubenstein JN, Hsieh MH, Chi T, Meng MV, Stoller ML. Failed percutaneous balloon dilation for renal access: incidence and risk factors. Urology. 2005;66(1):29-32.

7. Falahatkar S, Neiroomand H, Akbarpour M, Emadi SA, Khaki N. One-shot versus metal telescopic dilation technique for tract creation in percutaneous nephrolithotomy: comparison of safety and efficacy. Journal of endourology/Endourological Society. 23(4):615-8. 2009.

8. Pathak AS, Bellman GC. One-step percutaneous nephrolithotomy sheath versus standard two-step technique. Urology. 2005;66(5):953-7.

9. Goharderakhshan RZ, Schwartz BF, Rudnick DM, Irby PB, Stoller ML. Radially expanding single-step nephrostomy tract dilator. Urology. 2001;58(5):693-6.

10. Jackman SV, Hedican SP, Peters CA, Docimo SG. Percutaneous nephrolithotomy in infants and preschool age children: experience with a new technique. Urology. 52(4):697-701. 1998.

LIMITATIONS: We feel the study is limited due to sample size so a study with a large sample size will be needed to confirm 


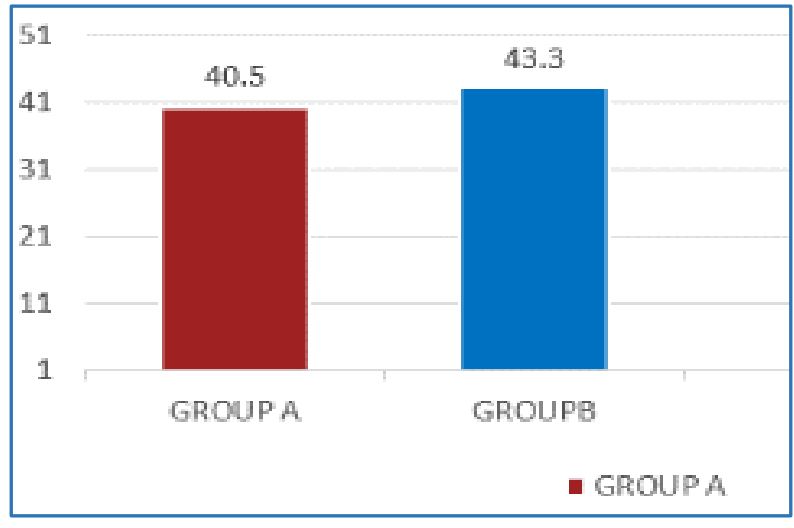

Fig. 1: Age Distribution

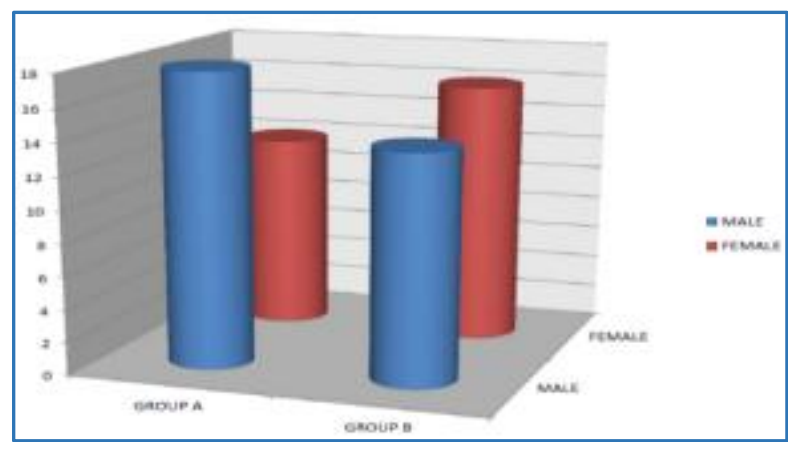

Fig. 2: Sex Distribution

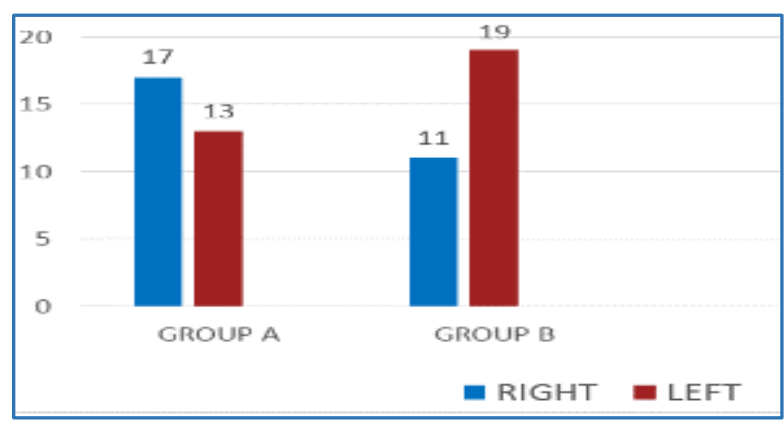

Fig. 3: Side Distribution

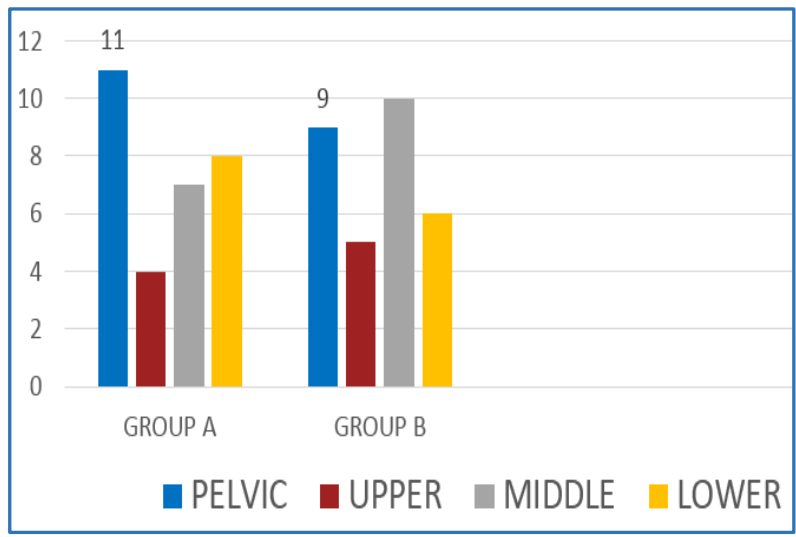

Fig. 4: Stone Distribution By Site

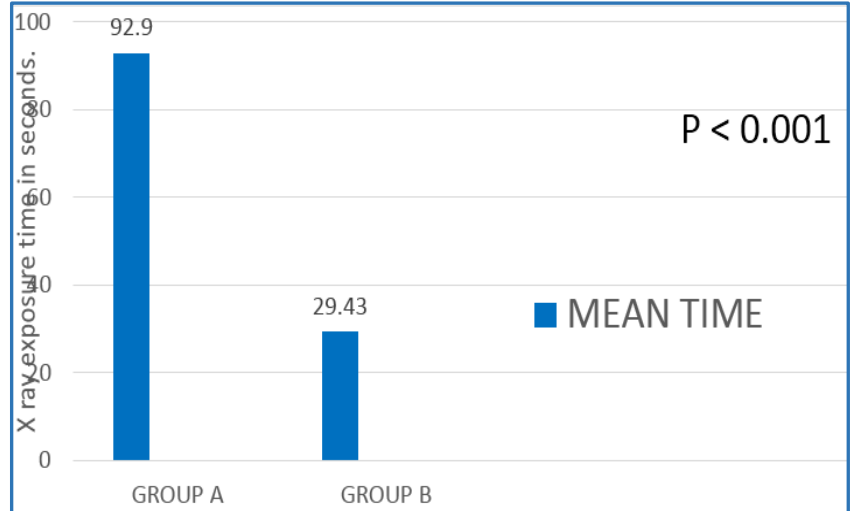

Fig. 5: Mean Xray Exposure Time In Seconds

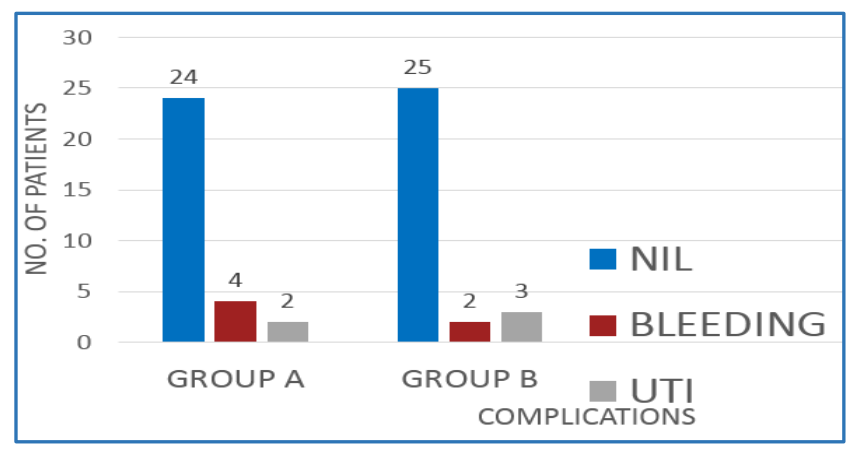

Fig. 6: Complications

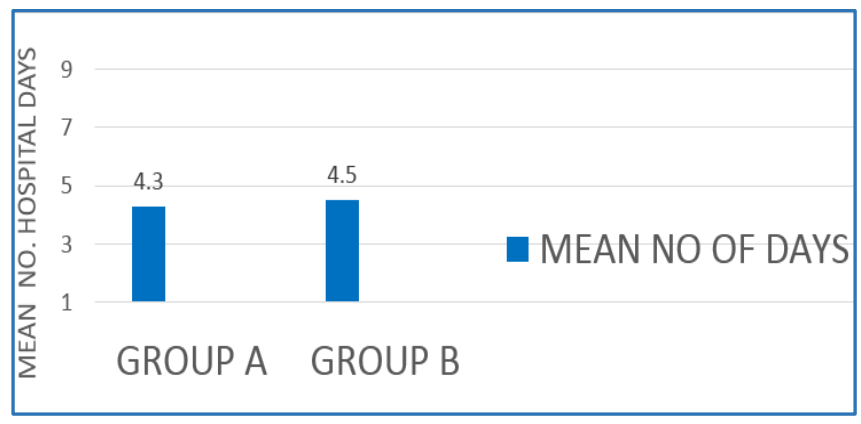

Fig. 7: Mean no of Days

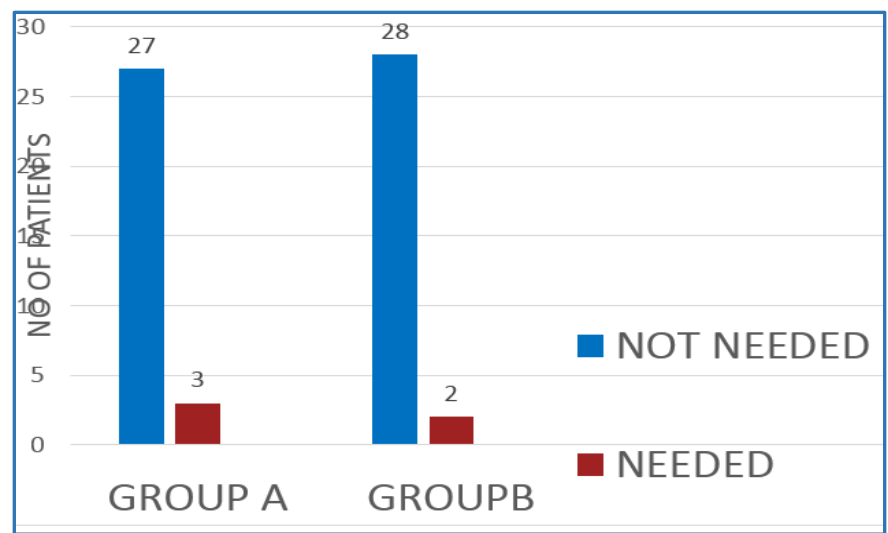

Fig. 8: Need For Eswl 